\title{
Evaluation of PRL-3 expression, and its correlation with angiogenesis and invasion in hepatocellular carcinoma
}

\author{
WEN-BO ZHAO, YING LI, XIN LIU, LING-YAN ZHANG and XIN WANG \\ Department of Hematology and Oncology, Provincial Hospital \\ Affiliated to Shandong University, Jinan 250021, P.R. China
}

Received March 6, 2008; Accepted May 2, 2008

DOI: 10.3892/ijmm_00000007

\begin{abstract}
Protein phosphatase of regenerating liver 3 (PRL-3) is a metastasis-associated phosphatase. Studies have shown that its overexpression increases cell motility and invasiveness. In this study, we aimed to investigate the expression of PRL-3 in hepatocellular carcinoma (HCC) tumor tissues and determine its correlations with matrix metalloproteinases (MMP-2, MMP-9) and E-cadherin in HCC. Paired cancerous and noncancerous tissues were freshly collected from 42 primary HCC patients. PRL-3 expression at both mRNA and protein level was evaluated by real-time PCR, Western blot analysis and immunohistochemistry. The microvessel density (MVD) in HCC was detected with immunohistochemistry. The mRNA expression of MMP-2, MMP-9 and E-cadherin was analyzed by real-time PCR in search of correlations with PRL-3. We found that PRL-3 was significantly up-regulated in the HCC tumor tissues compared with corresponding noncancerous liver tissues $(0.664 \pm 0.053$ vs. $0.024 \pm 0.003, \mathrm{P}<0.001)$. The mRNA level of PRL-3 in tissues was correlated with serum $\alpha$-fetoprotein level, vascular invasion and metastasis $(\mathrm{P}<0.001)$. PRL-3 expression was closely related to MVD. Furthermore, we found a significant correlation between PRL-3 mRNA expression and MMP-2, MMP-9 and E-cadherin. Our results demonstrated that PRL-3 is up-regulated in HCC. It is strongly suggested that PRL-3 plays a key role in the angiogenesis and invasion of HCC. MMP-2, MMP-9 and E-cadherin might be involved in PRL-3 functions in HCC.
\end{abstract}

\section{Introduction}

With over half a million new cases each year, hepatocellular carcinoma (HCC) is the fifth most common cancer worldwide and the third leading cause of cancer-related death (1). It is particularly prevalent in Asia and sub-Saharan Africa, yet

Correspondence to: Professor X. Wang, Department of Hematology and Oncology, Provincial Hospital Affiliated to Shandong University, Jinan 250021, P.R. China

E-mail:xin.wang55@yahoo.com.cn

Key words: hepatocellular carcinoma, phosphatase of regenerating liver 3, matrix metalloproteinase, E-cadherin, angiogenesis there has been a rising incidence and a progressive increase in HCC-related mortality in the United States and Western Europe $(2,3)$. HCC tends to show early invasion into blood vessels as well as intrahepatic metastasis (4). Despite advances in surgical and nonsurgical therapies, the outcome of HCC is poor. It is therefore crucial to understand the molecular mechanism of this highly aggressive cancer and to develop novel therapies for it.

Angiogenesis, which begins with the breakdown of surrounding basal membrane by activated endothelial cells, plays an important role in the early stage of multi-step hepatocarcinogenesis (5-7). Invasion through basement membrane and interstitial extracellular matrix is another key event for metastatic progression, which requires the action of a series of proteolytic enzymes, matrix metalloproteinases (MMPs). MMPs are a family of enzymes capable of degrading the constituents of the extracellular matrix and the basement membrane, thus mediating multiple pathologic processes including tumor angiogenesis and metastasis $(8,9)$. MMP-2 and MMP-9 are two members of the MMP family that are closely correlated with metastatic potential; both can degrade denatured collagens and type IV collagen present in the basement membrane (10). At the same time, the dispersion of tumor cells relies on the loss of E-cadherin, a cell adhesion molecule, which is involved in homotypic calcium-dependent cell-cell adhesion in epithelial tissues (11). Down-regulation of E-cadherin-mediated intercellular adhesion has increased tumor differentiation, invasion, metastasis and poor prognosis in human cancers including HCC (12-14).

Increasing evidence suggests that phosphatase of regenerating liver 3 (PRL-3) plays multiple roles in cancer metastasis. Its role in colorectal cancer metastasis and tumor-related angiogenesis has been identified (15). PRL-3 belongs to a subfamily of the protein tyrosine phosphatases (PTPs) with unique catalytic $\mathrm{COOH}$-terminal prenylation motif domain (16). This unique feature among PTPs also suggests that they may have distinct functions compared with other PTPs. In addition, all PRLs have significant sequence homology to dual-specific phosphatase PTEN and Cdc14 in regions other than the conserved PTP signature motif $(17,18)$. Compared to PRL-1 and PRL-2, the expression of PRL-3 is more distinct and frequent in cancer (19). PRL-3 was recently reported to be a molecular marker of tumor endothelial cells (20). It is found in a subset of endothelial cells in some human colon samples and expressed predominately in the vasculature of invasive breast cancer $(21,22)$. PRL-3 has been directly 
implicated in cancer progression. A high level of PRL-3 and increased invasion has been observed in the Hep3B HCC cell line (20). It has been suggested that PRL-3 might be a crucial effector in the MAPK cascade (23). Through this cascade, PRL-3 can enhance tumorigenesis and angiogenesis. It was shown that Chinese hamster ovary cells stably expressing PRL-3 exhibited enhanced motility and invasive activity, and induced metastatic tumor formation in nude mice (24). Further studies suggested that both PRL-3-expressing Chinese hamster ovary cells and PRL-3-expressing human DLD-1 colon cancer cells can redirect the migration of human umbilical vein endothelial cells (HUVEC) towards them, and PRL-3-expressing DLD-1 cells can enhance HUVEC vascular formation (25). These results indicate that PRL-3 contributes to the angiogenetic potential and invasive or metastatic properties of cancer cells. PRL-3 may be a driver for tumor supportive endothelial phenotypes.

However, the expression of PRL-3 in HCC has not been reported yet. To investigate the role and possible mechanism of PRL-3 in the carcinogenesis and the angiogenesis of HCC, the mRNA and protein expression of PRL-3 in tissues from HCC patients was analyzed. We also investigated the relation of PRL-3 expression to MMPs and E-cadherin in HCC.

\section{Materials and methods}

Patients and specimens. Tumor tissues and surrounding noncancerous hepatic parenchyma were freshly collected from 42 primary HCC patients who received resections at Shandong Provincial hospital during May 2005 to June 2007. The samples were from 34 males and 8 females with an average age of 54.5 years. Normal liver tissues from 15 patients with hepatorrhexis or liver hemangiomas were also obtained as controls. The clinical data of HCC patients are summarized in Table I. HCC was histologically diagnosed. The remainder was fixed in $4 \%$ paraformaldehyde, snap frozen in liquid nitrogen and stored at $-80^{\circ} \mathrm{C}$ for further use. Ethical approval was gained from the Health Service Ethics Committee of Shandong Provincial Hospital prior to commencement of the study. Written informed consent was obtained from the patients.

RNA extraction and cDNA synthesis. Total RNA was isolated from the liver tissues with TRIzol reagent (Invitrogen Corp., USA). The optical density at A260/280 nm of RNA was between 1.6 and 2.0. The integrity of RNA was confirmed by the presence of intact $18 \mathrm{~S}$ and $28 \mathrm{~S}$ bands on $1 \%$ agarose gel. The first-strand complementary DNA synthesis reaction was performed with MBI Fermentas Reverse Transcription Kit (MBI Fermentas, Vilnius, Lithuania) according to the manufacturer's instructions. The reaction was conducted at $42^{\circ} \mathrm{C}$ for $60 \mathrm{~min}$ and then at $70^{\circ} \mathrm{C}$ for $10 \mathrm{~min}$.

Real-time PCR. The primer sequences for real-time quantitative PCR analysis were designed and synthesized as follows: PRL-3 forward 5'-CACGCTCAGCACCTTCATTG-3', reverse 5'-GGTGAGCTGCTTGCTGTTGA-3'; MMP-2 forward 5'-CACCTACTGAGTGGCCGTGTT-3', reverse 5'-CAGGGAGCAGAGATTCGGAC-3'; MMP-9 forward 5'-TGCCTGCAACGTGAACATCT-3', reverse 5'-CACTT
GTCGGCGATAAGGAA-3'; E-cadherin forward 5'-CTC GGCCTGAAGTGACTCGT-3', reverse 5'-GGGTCAGTA TCAGCCGCTTT-3'; ß-actin forward 5'-AATGCTTCTAG GCGGACTATGA-3', reverse 5'-CAAGAAAGGGTGTAA CGCAACT-3'. For quantification the Quantitative SYBR Green PCR Kit (Tiangen, Beijing, P.R. China) and ABI Prism 7000 (ABI, USA) Sequence Detection System were used in real-time PCR analysis of the gene expression levels of PRL-3, MMP-2, MMP-9 and E-cadherin under the following conditions: 1 cycle at $95^{\circ} \mathrm{C}$ for $5 \mathrm{~min}$, then 30 cycles at $94^{\circ} \mathrm{C}$ for $30 \mathrm{sec}$ and $60^{\circ} \mathrm{C}$ for $45 \mathrm{sec}$; quantitative RT-PCR was repeated at least 3 times. The expression of $\beta$-actin was quantified to standardize the amount of RNA as an inner control. The mRNA in each sample was automatically quantitated with reference to the standard curve conducted each time using ABI 7000 software.

Western blotting. Tissues were lysed by RIPA (Biocolor Biotech, Shanghai, P.R. China) with $10 \mu 1$ PMSF on ice. The protein concentration was determined with a BCA Protein Assay Kit (Biocolor Biotech). Proteins suspended in loading buffer were heated at $100^{\circ} \mathrm{C}$ for $5 \mathrm{~min}$ for denaturation, separated in $15 \%$ SDS-PAGE and electrophoretically transferred onto PVDF membrane (Millipore, Bedford, MA) in transfer buffer at $40 \mathrm{~V}$ for $105 \mathrm{~min}$. The membrane was blocked with $5 \%$ defatted milk powder for $2 \mathrm{~h}$, washed with TBST and incubated with mouse anti-PRL-3 antibody (dilution 1:1000, R\&D Systems, Minneapolis, MN) overnight at $4{ }^{\circ} \mathrm{C}$. This was followed by incubation with an anti-mouse horseradish peroxidase-conjugated secondary antibody (Dako, Glostrup, Denmark) at a dilution of 1:200 at room temperature for $1 \mathrm{~h}$. Protein bands were visualized by SuperSignal West Pico Chemiluminescent Substrate kit (Pierce, Rockford, IL) and exposed with Kodak X-Ray film (Kodak, Rochester, NY). Proteins were re-blotted with anti- $\beta$-actin (diluted 1:10000, Zymed Laboratories, Inc., South San Francisco, CA) as an internal control.

Immunohistochemical staining and microvessel density $(M V D)$ detection. For immunohistochemical analysis, 4- $\mu \mathrm{m}$ sections were cut from paraffin blocks and baked at $50-60^{\circ} \mathrm{C}$ for at least $2 \mathrm{~h}$ before being stained with the following primary antibodies: rabbit anti-PRL-3 (dilution 1:100, Zymed Laboratories), mouse anti-CD34 (diluted 1:50; Santa Cruz, USA). The paraffin sections were dewaxed with xylene and rehydrated through a graded alcohol series. Endogenous peroxidase activity was blocked with $3 \% \mathrm{H}_{2} \mathrm{O}_{2}$ in methanol for $30 \mathrm{~min}$. Tissue sections were then pretreated in citrate buffer using a water bath for 15 min for antigen retrieval. One percent goat serum was applied to the sections to prevent nonspecific binding. For negative controls, primary antibody was replaced using PBS. Primary antibody was applied at $37^{\circ} \mathrm{C}$ for $2 \mathrm{~h}$ or overnight at $4^{\circ} \mathrm{C}$ in a humidified chamber. Anti-rabbit or mouse antibody kit (Jing Mei Biotech, Shanghai, P.R. China) was applied and $\mathrm{ABC}$ reaction was performed. Histomorphometric analysis was performed with the ImagePro Plus image analysis system (Media Cybernetics, Inc., USA).

For a count of microvessels, the four most highly vascularized areas were counted in x200 magnification fields and 
Table I. The correlations between PRL-3 mRNA expression and clinicopathological parameters in 42 patients with HCC.

\begin{tabular}{|c|c|c|c|}
\hline $\begin{array}{l}\text { Clinicopathological } \\
\text { parameters }\end{array}$ & Cases & $\begin{array}{c}\mathrm{T} / \mathrm{N}^{\mathrm{a}} \\
(\mathrm{mean} \pm \mathrm{SE})\end{array}$ & P-value \\
\hline \multicolumn{4}{|l|}{ Age } \\
\hline$<60$ years & 22 & $6.52 \pm 1.040$ & $0.598^{b}$ \\
\hline$\geq 60$ years & 20 & $7.83 \pm 1.250$ & \\
\hline \multicolumn{4}{|l|}{ Gender } \\
\hline Male & 34 & $7.03 \pm 0.599$ & $0.452^{\mathrm{b}}$ \\
\hline Female & 8 & $6.55 \pm 0.964$ & \\
\hline \multicolumn{4}{|l|}{ Virus } \\
\hline $\mathrm{HBV}$ & 30 & $7.28 \pm 0.856$ & $0.067^{\mathrm{c}}$ \\
\hline $\mathrm{HCV}$ & 2 & $4.52 \pm 0.794$ & \\
\hline None & 10 & & \\
\hline \multicolumn{4}{|l|}{ AFP } \\
\hline$<20 \mathrm{ng} / \mathrm{ml}$ & 16 & $3.45 \pm 0.680$ & $<0.001^{\mathrm{b}}$ \\
\hline$\geq 20 \mathrm{ng} / \mathrm{ml}$ & 26 & $8.48 \pm 0.711$ & \\
\hline \multicolumn{4}{|l|}{ Tumor multiplicity } \\
\hline Solitary & 38 & $7.62 \pm 0.655$ & $0.744^{b}$ \\
\hline Multiple & 4 & $6.59 \pm 0.832$ & \\
\hline \multicolumn{4}{|l|}{ Tumor size } \\
\hline$<3.5 \mathrm{~cm}$ & 14 & $6.52 \pm 0.580$ & $0.211^{\mathrm{b}}$ \\
\hline$\geq 3.5 \mathrm{~cm}$ & 28 & $7.55 \pm 0.820$ & \\
\hline \multicolumn{4}{|l|}{ Histological type of tumor } \\
\hline Well differentiated & 7 & $4.89 \pm 1.230$ & $0.289^{c}$ \\
\hline Moderately differentiated & 33 & $6.98 \pm 0.930$ & \\
\hline Poorly differentiated & 2 & $4.46 \pm 0.850$ & \\
\hline \multicolumn{4}{|l|}{ Liver cirrhosis } \\
\hline Present & 25 & $7.48 \pm 0.950$ & $0.478^{\mathrm{b}}$ \\
\hline Absent & 17 & $6.82 \pm 1.030$ & \\
\hline \multicolumn{4}{|l|}{ Vascular invasion } \\
\hline Present & 13 & $7.08 \pm 1.260$ & $0.001^{\mathrm{b}}$ \\
\hline Absent & 29 & $4.97 \pm 0.830$ & \\
\hline
\end{tabular}

${ }^{a} \mathrm{~T} / \mathrm{N}$ ratio: $\mathrm{PRL}-3 / \mathrm{B}$-actin in tumor $(\mathrm{T})$ divided by PRL-3/ß-actin in noncancerous tissue $(\mathrm{N})$; ${ }^{\mathrm{b}} \mathrm{P}$-value determined by the Mann-Whitney $\mathrm{U}$ test. ${ }^{\mathrm{C}} \mathrm{P}$-value determined by the Kruskal-Wallis test. HBV, hepatitis B virus; HCV, hepatitis C virus; AFP, $\alpha$-fetoprotein; SE, standard error.

the average counts were then recorded. The intensity of cytoplasmic staining for PRL-3 was graded as follows: no staining or staining observed in $<10 \%$ of tumor cells, 0 ; faint/barely perceptible staining detected in $\geq 10 \%$ of tumor cells, $1+$; a moderate or strong complete staining observed in $\geq 10 \%$ of tumor cells, $2+$ or $3+$, respectively. A score of 0 or $1+$ was considered negative, whereas scores of $2+$ or $3+$ were considered positive. The result was graded independently by two pathologists at different times. The grading results were further verified using the automated Chromavision Cellular Imaging System (Chromavision Medical Systems, Inc., San Juan Capistrano, CA).

Statistical analysis. The unpaired Student's t-test was used to analyze the differences of PRL-3 mRNA expression in HCC

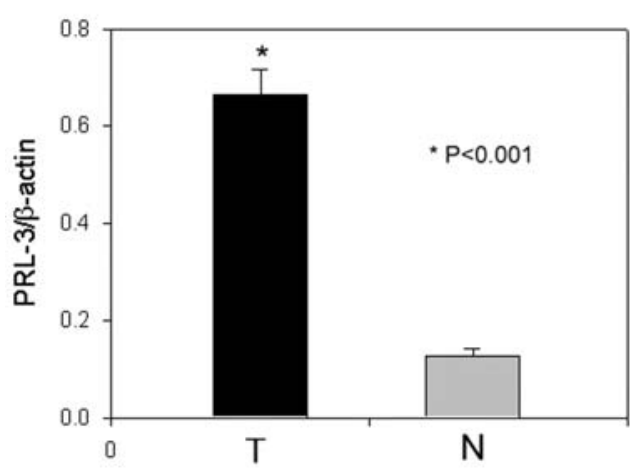

Figure 1. Real-time PCR of PRL-3 in HCC tissues. Expression levels were normalized to that of $\beta$-actin. The relative PRL-3 mRNA expression of PRL-3 in HCC tissues was significantly higher than in noncancerous counterparts $(0.664 \pm 0.053$ vs. $0.024 \pm 0.003, \mathrm{P}<0.001)$.

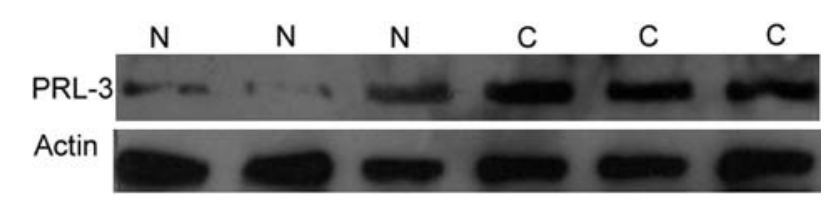

Figure 2. Western blot analysis of PRL-3 expression in HCC cancerous tissues and noncancerous liver tissues. A significantly higher expression of PRL-3 at protein level is visible. N, noncancerous liver tissues; C, HCC cancerous tissues.

tissues compared to noncancerous liver tissues. To analyze the correlation of mRNA expression of PRL-3 with clinicopathological parameters, the Mann-Whitney $U$ and Kruskal-Wallis tests were used. Correlations of mRNA expression levels were analyzed with Pearson's test. $\mathrm{P}<0.05$ was considered statistically significant. Data were analyzed using SPSS 11.5 (SPSS, Inc., IL).

\section{Results}

Quantitative $m R N A$ expression of $P R L-3$ in $H C C$. We examined $42 \mathrm{HCC}$ samples and corresponding noncancerous hepatic tissues for PRL-3 mRNA expression using real-time quantitative RT-PCR. The expression of PRL-3 in HCC cancerous tissues showed a noticeable up-regulation in comparison with noncancerous liver tissues, as shown in Fig. 1. The average PRL-3/ß-actin level in HCC tissues is significantly higher than in noncancerous liver $(0.664 \pm 0.053$ vs. $0.127 \pm 0.015, \mathrm{P}<0.001$ ) (Fig. 1).

Correlation of PRL-3 mRNA expression and clinical parameters of HCC patients. The correlation between PRL-3 mRNA expression and clinicopathological parameters of the 42 HCC patients, was statistically analyzed and is listed in Table I. There was a significant correlation between the high expression of PRL-3 and high serum $\alpha$-fetoprotein (AFP) levels and vascular invasion $(\mathrm{P}<0.05)$. PRL-3 expression was increased in HCC with liver cirrhosis. However, the difference was not statistically significant. No significant correlation was found between PRL-3 expression and age, gender, tumor size, tumor multiplicity and differentiaion. 

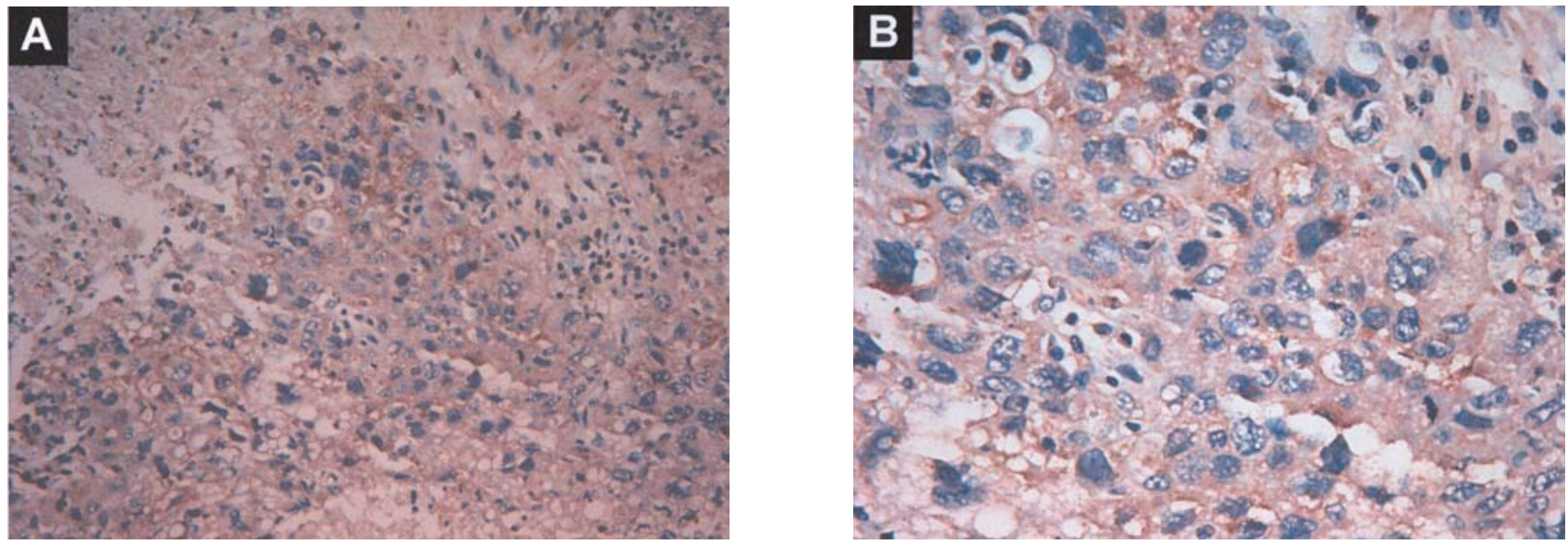

Figure 3. Immunohistochemical staining of PRL-3 in HCC tumor tissues using anti-PRL-3 antibodies. Positive staining was diffused in the cytoplasm of the cancer cells. Original magnification x200 (A); x400 (B).
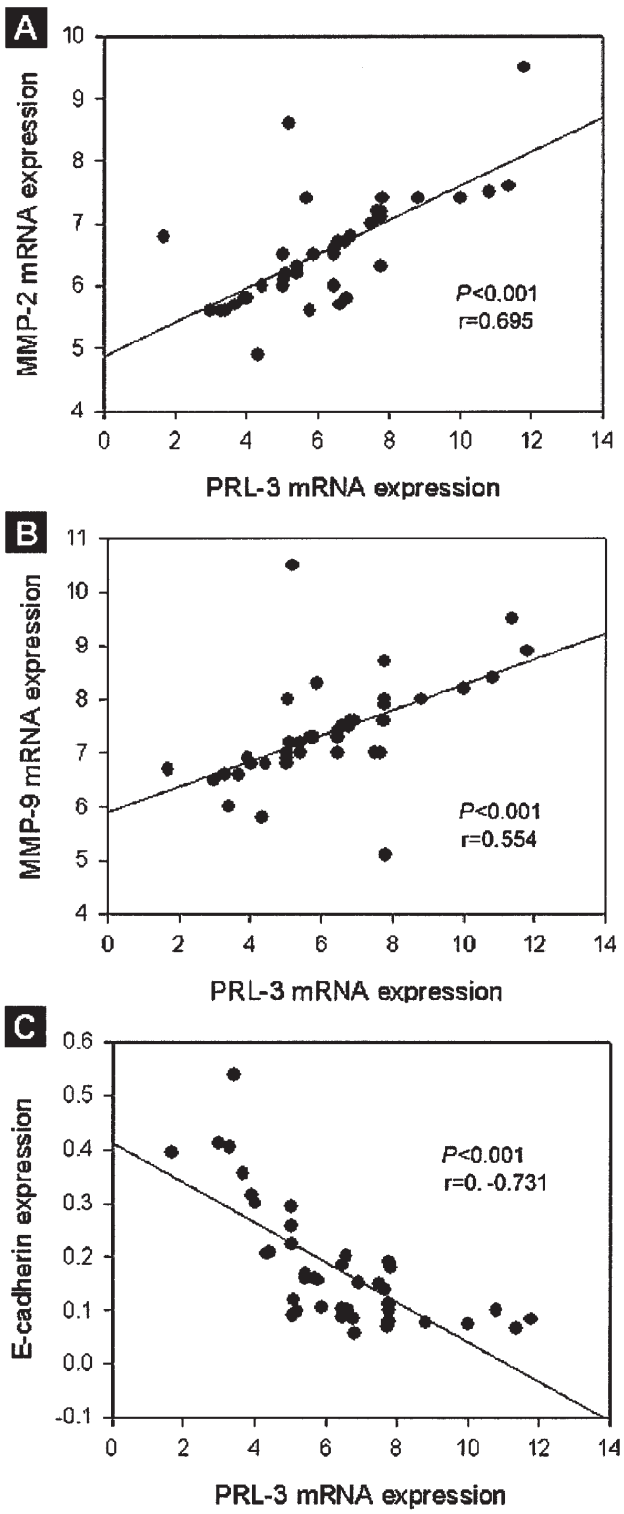

Figure 4. Correlations of PRL-3 mRNA expression and MMP-2, MMP-9, and E-cadherin. (A) A significant correlation between the mRNA expression of PRL-3 and that of MMP-2 was observed ( $r=0.695, \mathrm{P}<0.001)$. (B) The PRL-3 and MMP-9 mRNA expression showed a significant positive correlation $(\mathrm{r}=0.554, \mathrm{P}<0.001)$. (C) The PRL-3 and E-cadherin mRNA expression demonstrated a significant negative correlation $(r=-0 .-0.731, \mathrm{P}<0.001)$.
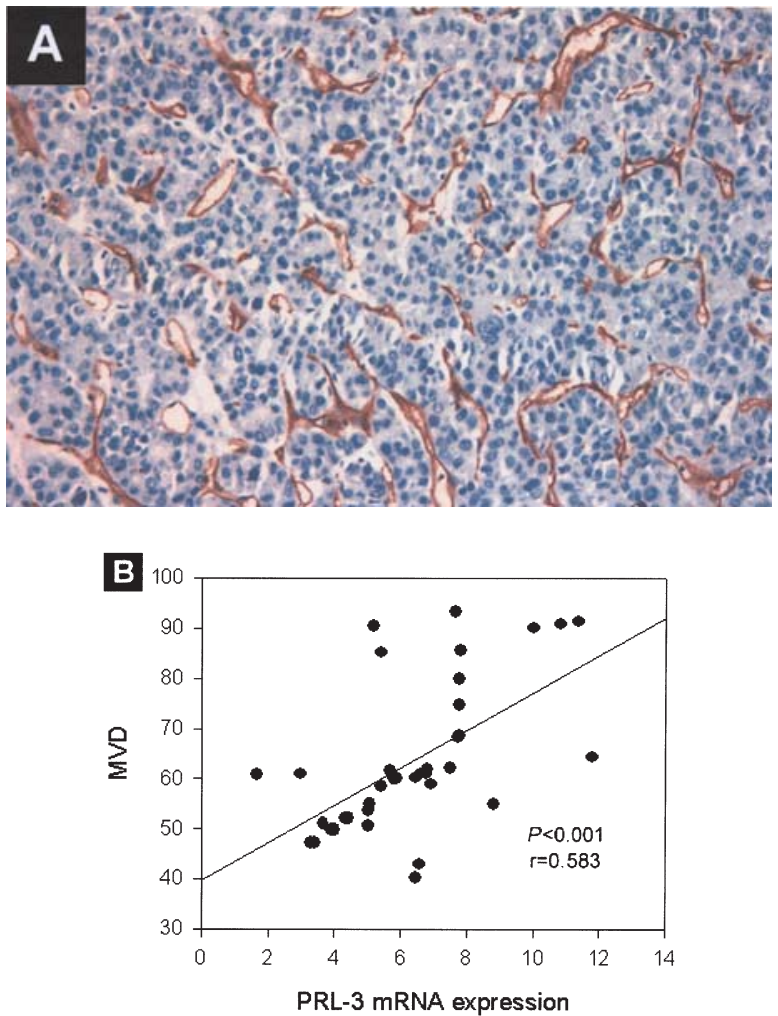

Figure 5. (A) Intensive staining of microvessels by anti-CD34 immunostaining in HCC (original magnification x200). (B) The PRL-3 mRNA expression and intratumoral MVD showed a highly significant positive correlation $(\mathrm{r}=0.583, \mathrm{P}<0.001)$.

Protein expression of $P R L-3$. Western blot analysis was conducted on the HCC tumor and corresponding noncancerous liver tissues. PRL-3 expression was up-regulated in HCC tissues HCC, but there was only a low expression in noncancerous hepatic tissues (see Fig. 2).

Immunohistochemical staining with a rabbit anti-PRL-3 antibody was performed. PRL-3 was expressed 57.27 and $28.73 \%$ in HCC and noncancerous tissue specimen respectively, on average. PRL-3 was localized mainly in the cytoplasm and was significantly elevated in HCC tissues. Notably, PRL-3 staining was found in the microvessels of HCC tissues (Fig. 3). 
Correlations of MMP-2, MMP-9, E-cadherin and PRL-3 expression in HCC. MMP-2, MMP-9 and E-cadherin expression in $\mathrm{HCC}$ tissues was also investigated for mRNA expression. Compared with noncancerous liver tissues, significantly increased expression of MMP-2 and MMP-9, and decreased expression of E-cadherin were found in HCC tissues. We found significant positive correlations between PRL-3 and MMP-2 ( $\mathrm{r}=0.695, \mathrm{P}<0.001)$ or MMP-9 ( $\mathrm{r}=0.554$, $\mathrm{P}<0.001)$. Furthermore, a significant negative correlation was also found between the mRNA expression of PRL-3 and E-cadherin ( $\mathrm{r}=-0 .-0.731, \mathrm{P}<0.001)$ (Fig. 4).

Correlation between PRL-3 mRNA expression and microvessel density (MVD) in HCC patients. Specific staining of microvessels by anti-CD34 antibodies was observed in the vessels and MVD was evaluated. Any brown-stained endothelial cell cluster distinct from adjacent microvessels, tumor cells, or other stromal cells was considered as a single countable microvessel. The most vascular areas of tumors were identified on a low-power field (x200), and vessels were counted in five high-power fields (x200). The median MVD in HCC was $66.7 \pm 11.2 /$ field and 11.6 $\pm 5.2 /$ field in noncancerous liver tissues. Significant correlations between the mRNA level of PRL-3 and MVD were observed ( $\mathrm{r}=0.583$, $\mathrm{P}<0.001$, Fig. 5).

\section{Discussion}

Hepatocellular carcinoma is multifactoral and complex in pathogenesis. Tumor angiogenesis and invasion play an important role in HCC progression and are predictors for poor prognosis (26). Anti-angiogenesis strategies are mainly focused on endothelial cell proliferation, angiogenic factors, and the degradation of extracellular matrix and basement membrane. Our study demonstrates a significantly high PRL-3 expression level in HCC tumor tissues, which is in close correlation with that of MMP-2, MMP-9 and E-cadherin in HCC. These results suggest that PRL-3 is involved in the angiogenic and invasive procession of $\mathrm{HCC}$, and therefore may be associated with the pathogenesis of HCC.

We detected PRL-3 mRNA expression in HCC tissues by quantitative PCR and found that PRL-3 mRNA was significantly up-regulated in human HCC tissues in comparison to corresponding noncancerous liver tissues. We also investigated the correlations between PRL-3 expression and clinicopathological parameters in HCC. A close relation was found between the mRNA expression of PRL-3 and serum AFP level and the metastasis of tumors. Serum AFP level was previously confirmed as a significant prognostic factor for HCC (27). Vascular invasion indicated as invasive phenotypes in $\mathrm{HCC}$ (28), and tends to be more frequent in patients with higher PRL-3 mRNA expression than those with a low level of PRL-3 expression. However, no significance was found in relation to gender, age, tumor multiplicity, and TNM stage. Further examination by Western blot analysis and immunohistochemical staining confirmed the high level of PRL-3 in HCC.

In this study, the mRNA expression of MMP-2, MMP-9 and E-cadherin in HCC tissues was assayed with quantitative real-time PCR. MMP levels were found to directly associate with the carcinogenesis, angiogenesis and invasion of hepatocellular carcinoma (10). The degradation of matrix protein allows cancer cells to invade the surrounding tissue. In experimental animal models, MMP-2 and MMP-9 activity have been directly associated with tumor angiogenic potential $(29,30)$. The close correlation of PRL-3 and either MMP-2 or MMP-9 implicated that PRL-3 might be involved in the secretion of MMPs.

E-cadherin is a cell-cell adhesion molecule and tumor invasion suppressor gene that is frequently altered in human cancers. The loss of E-cadherin results in the disassembly of cell-cell adhesion junctions and increases tumor cell invasiveness in vitro. Studies have revealed that the loss of E-cadherin expression is closely related to hepatocellular carcinoma progression from low to high histological grade and further correlate it with vascular invasion and metastasis (31). Our study revealed a decreased expression of E-cadherin in HCC tissues and a close relation of PRL-3 and E-cadherin. Therefore, it can be inferred that PRL-3 might be involved in triggering angiogenesis and establishing microvasculature in HCC, and also involved in the spread of the tumor.

It has been reported that PRL-3 can stimulate Rho family GTPase signaling pathways which are critical regulators of actin organization to promote cell motility and invasion (32). PRL-3 promoted the activation of the Rho family GTPases RhoA and RhoC, and reduced the activity of Rac (33). However, Rac activity has been reported to promote E-cadherin mediated cell-cell adherence and therefore antagonize cell invasiveness (34). This suggests that PRL-3 might contribute to the decrease of E-cadherin expression in HCC. Furthermore, the up-regulation of PRL-3 activates Src kinase, which initiates a number of signal pathways culminating in the phosphorylation of ERK1/2, STAT3, and p130 (Cas) through down-regulation of Csk, a negative regulator of Src (35). Additionally, induction of MMP-2 and MMP-9 expression can be mediated by ERK1/2 and PI3K/Akt pathways, in spite of the differences of the expression style $(36,37)$. We hypothesize that cancer cells are activated with a higher expression level of PRL-3, resulting in higher migration and invasion ability. Simultaneously, a series of signaling pathways in tumor cells may be activated and involved in the progression of HCC, through the up-regulation of MMPs or down-regulation of E-cadherin.

In conclusion, PRL-3 was up-regulated in HCC tissues and PRL-3 mRNA expression was significantly correlated to the CD34-MVD and closely associated with the expression of MMP-2, MMP-9 and E-cadherin in tumor tissues. Detection of high PRL-3 expression in HCC tissues might assist in identifying high-risk patients in need of further therapy. Dephosphorylation events due to PRL-3 activity are closely associated with HCC, suggesting that selective PRL-3 inhibitors might have therapeutic value and effectively block the angiogenesis and invasion procession of HCC.

\section{Acknowledgements}

We thank the medical and nursing staff in the Hepatobiliary Surgery department at Shandong Provincial Hospital for providing clinical samples. We also thank Professor Li You and Jie Zhang for excellent technical assistance. This work 
was supported by the Key Scientific \& Technological Project of Shandong Province (No. 2006GG2202007)

\section{References}

1. Parkin DM, Bray F, Ferlay J and Pisani P: Global cancer statistics, 2002. CA Cancer J Clin 55: 74-108, 2005.

2. El-Serag HB, Davila JA, Petersen NJ and McGlynn KA: The continuing increase in the incidence of hepatocellular carcinoma in the United States: an update. Ann Int Med 139: 817-823, 2003.

3. Parkin DM: Global cancer statistics in the year 2000. Lancet Oncol 2: 533-543, 2001

4. Ozaki I, Mizuta T, Zhao G, et al: Involvement of the Ets-1 gene in overexpression of matrilysin in human hepatocellular carcinoma. Cancer Res 60: 6519-6525, 2000.

5. Carmeliet P: Mechanisms of angiogenesis and arteriogenesis. Nature Medicine 6: 389-395, 2000.

6. Park YN, Kim YB, Yang KM and Park C: Increased expression of vascular endothelial growth factor and angiogenesis in the early stage of multistep hepatocarcinogenesis. Arch Pathol Lab Med 124: 1061-1065, 2000.

7. Croix BS, Rago C, Velculescu V, et al: Genes expressed in human tumor endothelium. Science 289: 1197-1202, 2000.

8. Nelson AR, Fingleton B, Rothenberg ML and Matrisian LM: Matrix metalloproteinases: biologic activity and clinical implications. J Clin Oncol 18: 1135-1149, 2000.

9. Moses MA: The regulation of neovascularization by matrix metalloproteinases and their inhibitors. Stem Cells 15: 180-189, 1997.

10. Egeblad M and Werb Z: New functions for the matrix metalloproteinases in cancer progression. Nat Rev Cancer 2: 161-174, 2002.

11. Wijnhoven BPL, Dinjens WNM and Pignatelli M: E-cadherincatenin cell-cell adhesion complex and human cancer. Br J Surg 87: 992-1005, 2000.

12. Endo K, Ueda T, Ueyama J, Ohta T and Terada T: Immunoreactive E-cadherin, alpha-catenin, beta-catenin, and gammacatenin proteins in hepatocellular carcinoma: Relationships with tumor grade, clinicopathologic parameters, and patients survival. Hum Pathol 31: 558-565, 2000.

13. Popov Z, de Medina SGD, Lefrere-Belda MA, et al: Low E-cadherin expression in bladder cancer at the transcriptional and protein level provides prognostic information. $\mathrm{Br} \mathrm{J}$ Cancer 83: 209-214, 2000.

14. Matsumura T, Makino R and Mitamura K: Frequent downregulation of E-cadherin by genetic and epigenetic changes in the malignant progression of hepatocellular carcinomas. Clin Cancer Res 7: 594-599, 2001.

15. Saha S, Bardelli A, Buckhaults P, et al: A phosphatase associated with metastasis of colorectal cancer. Science 294: $1343-1346,2001$

16. Guo K, Li J, Tang JP, Koh V, Gan BQ and Zeng Q: Catalytic domain of PRL-3 plays an essential role in tumor metastasis, formation of PRL-3 tumors inside the blood vessel. Cancer Biol Ther 3: 945-951, 2004.

17. Diamond RH, Cressman DE, Laz TM, Abrams CS and Taub R: PRL-1, a unique nuclear protein tyrosine phosphatase, affects cell growth. Mol Cell Biol 14: 3752-3762, 1994.

18. Zeng Q, Hong W and Tan YH: Mouse PRL-2 and PRL-3, two potentially prenylated protein tyrosine phosphatases homologous to PRL-1. Biochem Biophys Res Commun 244: 421-427, 1998 .
19. Stephens BJ, Han H, Gokhale V and Von Hoff DD: PRL phosphatases as potential molecular targets in cancer. Mol Cancer Ther 4: 1653-1661, 2005

20. Rouleau C, Roy A, St. Martin T, et al: Protein tyrosine phosphatase PRL-3 in malignant cells and endothelial cells: expression and function. Mol Cancer Ther 5: 219-229, 2006.

21. Bardelli A, Saha S, Sager JA, et al: PRL-3 expression in metastatic cancers. Clin Cancer Res 9: 5607-5615, 2003.

22. Parker BS, Argani P, Cook BP, et al: Alterations in vascular gene expression in invasive breast carcinoma. Cancer Res 64: 7857-7866, 2004.

23. Peng L, Jin G, Wang L, Guo J, Meng L and Shou C: Identification of integrin $\mathrm{a} 1$ as an interacting protein of protein tyrosine phosphatase PRL-3. Biochem Biophys Res Commun 342: 179-183, 2006

24. Zeng Q, Dong JM, Guo K, et al: PRL-3 and PRL-1 promote cell migration, invasion, and metastasis. Cancer Res 63: 2716-2722, 2003.

25. Guo K, Li J, Wang H, et al: PRL-3 Initiates tumor angiogenesis by recruiting endothelial cells in vitro and in vivo. Cancer Res 66: 9625-9635, 2006.

26. Llovet JM, Burroughs A and Bruix J: Hepatocellular carcinoma. Lancet 362: 1907-1917, 2003.

27. Ijichi M, Takayama T, Matsumura M, Shiratori Y, Omata M and Makuuchi M: $\alpha$-Fetoprotein mRNA in the circulation as a predictor of postsurgical recurrence of hepatocellular carcinoma: A prospective study. Hepatology 35: 853-860, 2002.

28. Poon RTP, Fan ST and Wong J: Risk factors, prevention, and management of postoperative recurrence after resection of hepatocellular carcinoma. Ann Surg 232: 10-24, 2000.

29. Fang JM, Shing Y, Wiederschain D, et al: Matrix metalloproteinase-2 is required for the switch to the angiogenic phenotype in a tumor model. Proc Natl Acad Sci USA 97: 3884-3889, 2000.

30. Bergers G, Brekken R, McMahon G, et al: Matrix metalloproteinase-9 triggers the angiogenic switch during carcinogenesis. Nat Cell Biol 2: 737-744, 2000.

31. Shimoyama Y and Hirohashi S: Cadherin intercellular adhesion molecule in hepatocellular carcinomas: loss of E-cadherin expression in an undifferentiated carcinoma. Cancer Lett 57: 131-135, 1991.

32. Sahai E and Marshall CJ: RHO-GTPases and cancer. Nat Rev Cancer 2: 133-142, 2002.

33. Fiordalisi JJ, Keller PJ and Cox AD: PRL Tyrosine phosphatases regulate Rho family GTPases to promote invasion and motility. Cancer Res 66: 3153-3161, 2006.

34. Hordijk PL, ten Klooster JP, van der Kammen RA, Michiels F, Oomen L and Collard JG: Inhibition of invasion of epithelial cells by Tiam1-Rac signaling. Science 278: 1464-1466, 1997.

35. Liang F, Liang J, Wang W-Q, Sun J-P, Udho E and Zhang Z-Y: PRL-3 promotes cell invasion and proliferation by downregulation of csk leading to Src activation. J Biol Chem 282: 5413-5419, 2007.

36. Chung TW, Lee YC and Kim CH: Hepatitis B viral HBx induces matrix metalloproteinase-9 gene expression through activation of ERKs and PI-3K/AKT pathways: Involvement of invasive potential. FASEB J 18: 1123-1125, 2004.

37. Park M-J, Kwak H-J, Lee H-C, et al: Nerve growth factor induces endothelial cell invasion and cord formation by promoting matrix metalloproteinase- 2 expression through the phosphatidylinositol 3-kinase/Akt signaling pathway and AP-2 transcription factor. J Biol Chem 282: 30485-30496, 2007. 